\title{
Long-range Hexagonal Pore Ordering as the Key to Controlling SERS Efficiency in Substrates Based on Porous Alumina
}

\author{
Mikhail Pashchanka,‡* Sherif Okeil‡ and Jörg J. Schneider
}

Fachbereich Chemie, Eduard-Zintl-Institut, Fachgebiet Anorganische Chemie, Technische Universität Darmstadt, Alarich-Weiss-Straße 12, 64287, Darmstadt, Germany. E-Mail: mikhail.pashchanka@gmail.com, pashchanka@ac.chemie.tu-darmstadt.de

$\ddagger$ These authors have contributed equally.

\section{Complete summary displaying:}

- Scanning electron microscopy (SEM) survey of the porous anodic alumina (PAA) samples obtained after different pre-anodizing periods (0.5-30 hours). Main anodizing lasted 5 minutes in all cases.

- Coherent domain sizes surface area calculations (data obtained using Gauss's area formula).

- Final statistical calculations results of the average long-range ordered domain sizes after data refinement and excluding of small short-range ordered domains from treatment.

Cyan areas in the color-coded SEM images correspond to the hexagonally ordered domains where every cell is coordinated with 6 nearest neighbors. Red areas correspond to disordered fields with random pore coordination numbers.

Dataset sizes used for statistical calculations of mean domain surface areas were as follows:

For the samples obtained from $0.3 \mathrm{M}$ oxalic acid electrolyte:

before the short-range ordered domains exclusion: 31-72 individual values from a randomly selected area after dataset refinement: $23-48$ individual values

For the samples obtained from 0.3 M sulfuric acid electrolyte:

before the short-range ordered domains exclusion: 25-64 individual values from a randomly selected area after dataset refinement: $14-25$ individual values

Dataset sizes obtained after the multiple steps of analysis and refinement are conditioned by the laboriousness of the employed method. However, a dataset size as small as 6 values is often required in practice to avoid any significant broadening of a confidence interval. Thus, the datasets used in this work are sufficiently large to be considered as representative for statistical calculations.

All confidence interval calculations were performed using the significance level 0.05 , which means that the domains of all possible sizes from a single analyzed PAA sample are contained within such interval with $95 \%$ probability. This leads to the broadening of confidence intervals for the PAA samples which are characterized by a significant actual spread in domain size values. This broadening is fully conditioned by sample properties and should not be considered as inaccuracy. 
Selection frame shows domains displayed only in part because of their intersections with SEM image margins. Such small visible fragments do not figure in statistical calculations. If the frequent use of large remaining fragments was unavoidable, a slight shift of the mean values is possible.

Series of experiments with $0.3 \mathrm{M}$ sulfuric acid electrolyte, $27 \mathrm{~V}, 0-0.5^{\circ} \mathrm{C}$ (8 different samples) Threshold used in all cases for dataset refinement and small domains elimination from statistics was $55000 \mathrm{~nm}^{2}$ (approximately corresponds to a symmetrical domain which contains 16 hexagonal cells).

Sample $10.3 \mathrm{M}$ sulfuric acid, $27 \mathrm{~V}, 0-0.5^{\circ} \mathrm{C}$, pre-anodizing duration: 0.5 hours

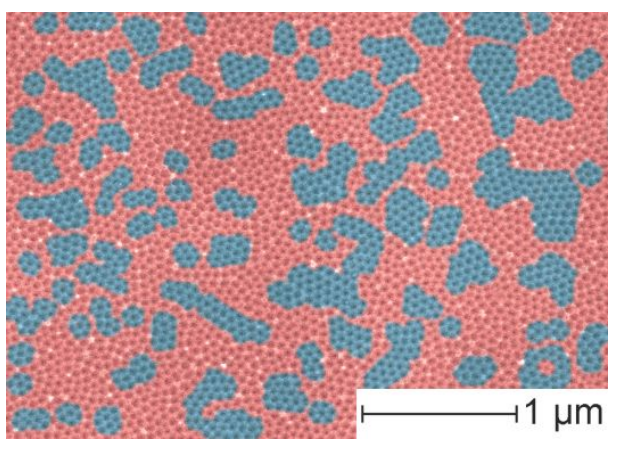

Mean long-range ordered domain size value: $107113 \mathrm{~nm}^{2}$

Standard deviation:

$55077 \mathrm{~nm}^{2}$

Half-width of the confidence interval:

$26182 \mathrm{~nm}^{2}$

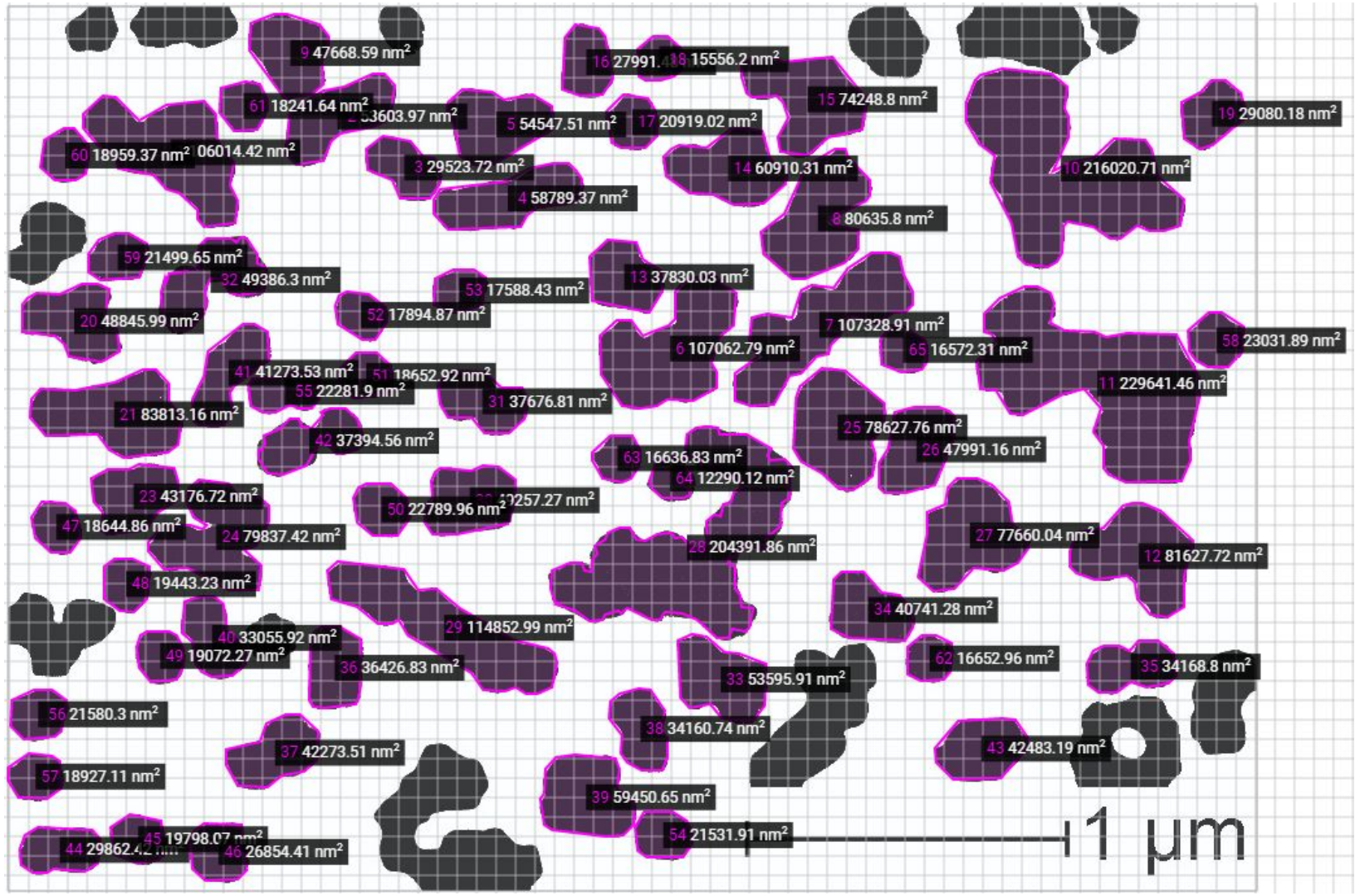


Sample $20.3 \mathrm{M}$ sulfuric acid, $27 \mathrm{~V}, 0-0.5^{\circ} \mathrm{C}$, pre-anodizing duration: 1 hour

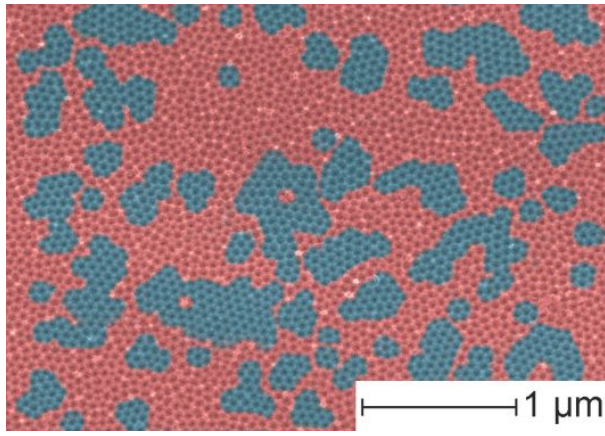

$\begin{array}{ll}\text { Mean long-range ordered domain size value: } & 130715 \mathrm{~nm}^{2} \\ \text { Standard deviation: } & 79514 \mathrm{~nm}^{2} \\ \text { Half-width of the confidence interval: } & 34008 \mathrm{~nm}^{2}\end{array}$

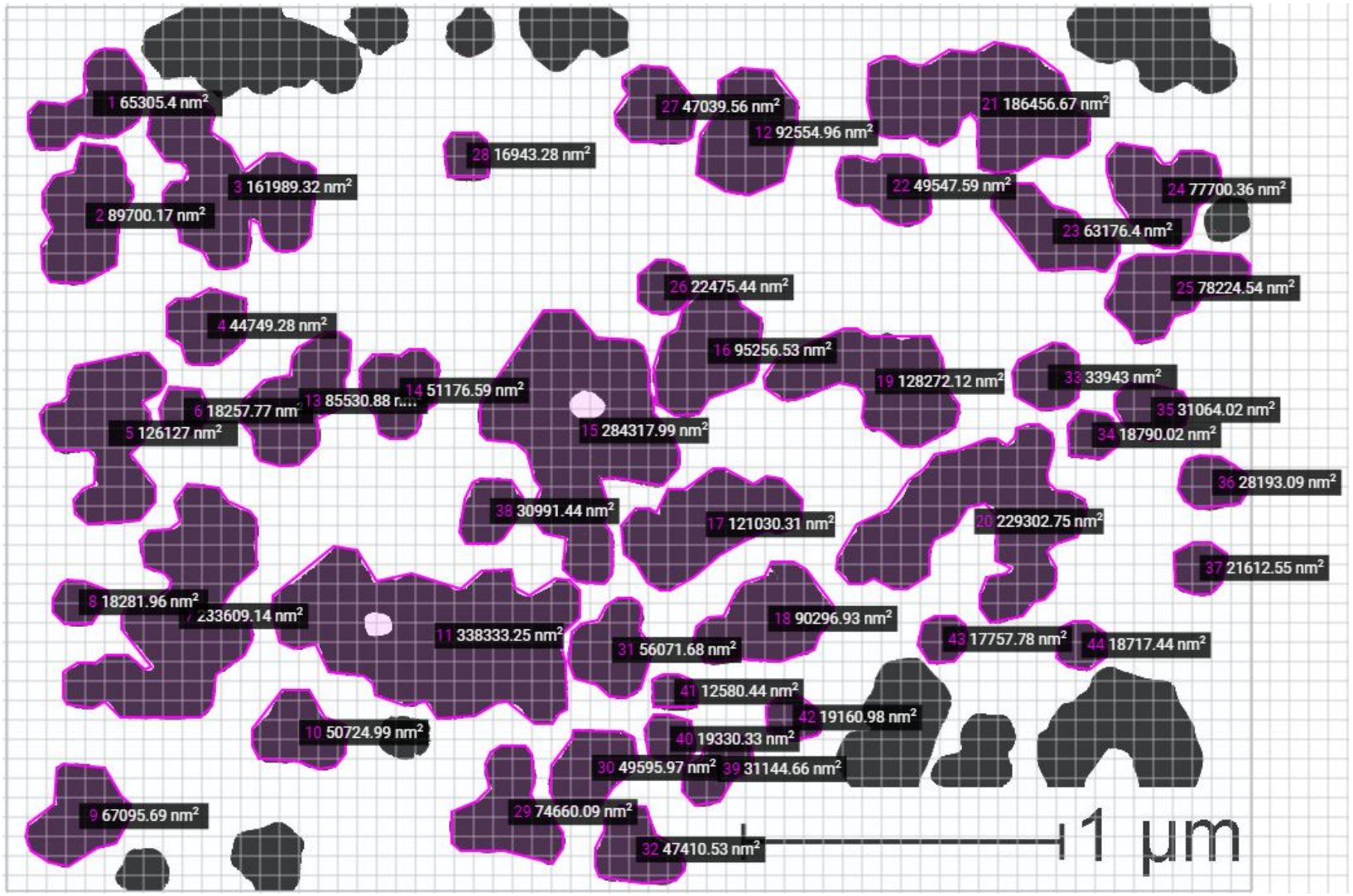


Sample $30.3 \mathrm{M}$ sulfuric acid, $27 \mathrm{~V}, 0-0.5^{\circ} \mathrm{C}$, pre-anodizing duration: 5 hours

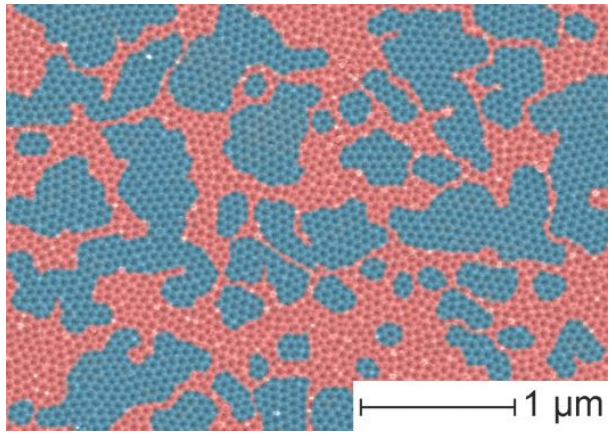

Mean long-range ordered domain size value: $\quad 207707 \mathrm{~nm}^{2}$

Standard deviation:

$198399 \mathrm{~nm}^{2}$

Half-width of the confidence interval:

$103926 \mathrm{~nm}^{2}$

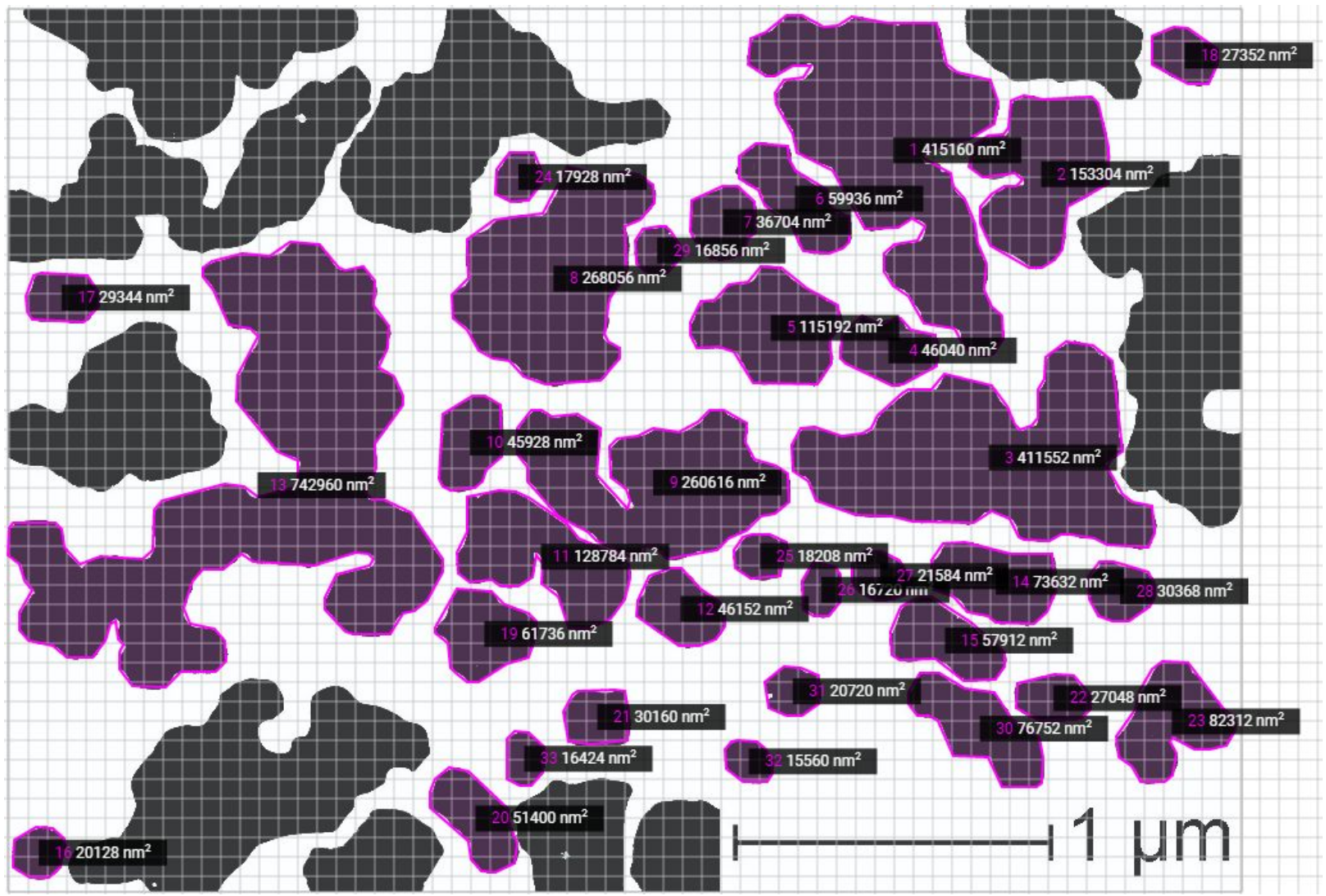


Sample $40.3 \mathrm{M}$ sulfuric acid, $27 \mathrm{~V}, 0-0.5^{\circ} \mathrm{C}$, pre-anodizing duration: 10 hours

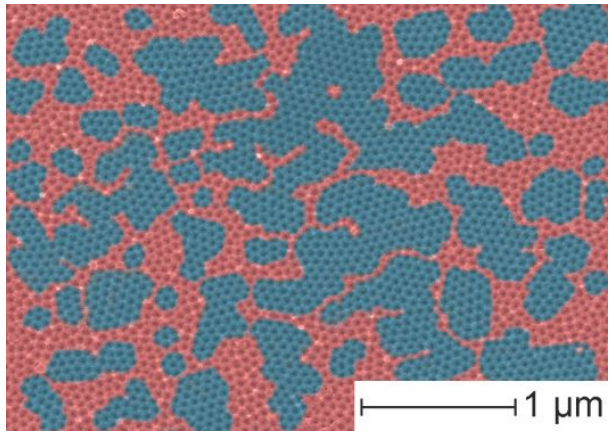

$\begin{array}{lr}\text { Mean long-range ordered domain size value: } & 225303 \mathrm{~nm}^{2} \\ \text { Standard deviation: } & 243610 \mathrm{~nm}^{2} \\ \text { Half-width of the confidence interval: } & 104192 \mathrm{~nm}^{2}\end{array}$

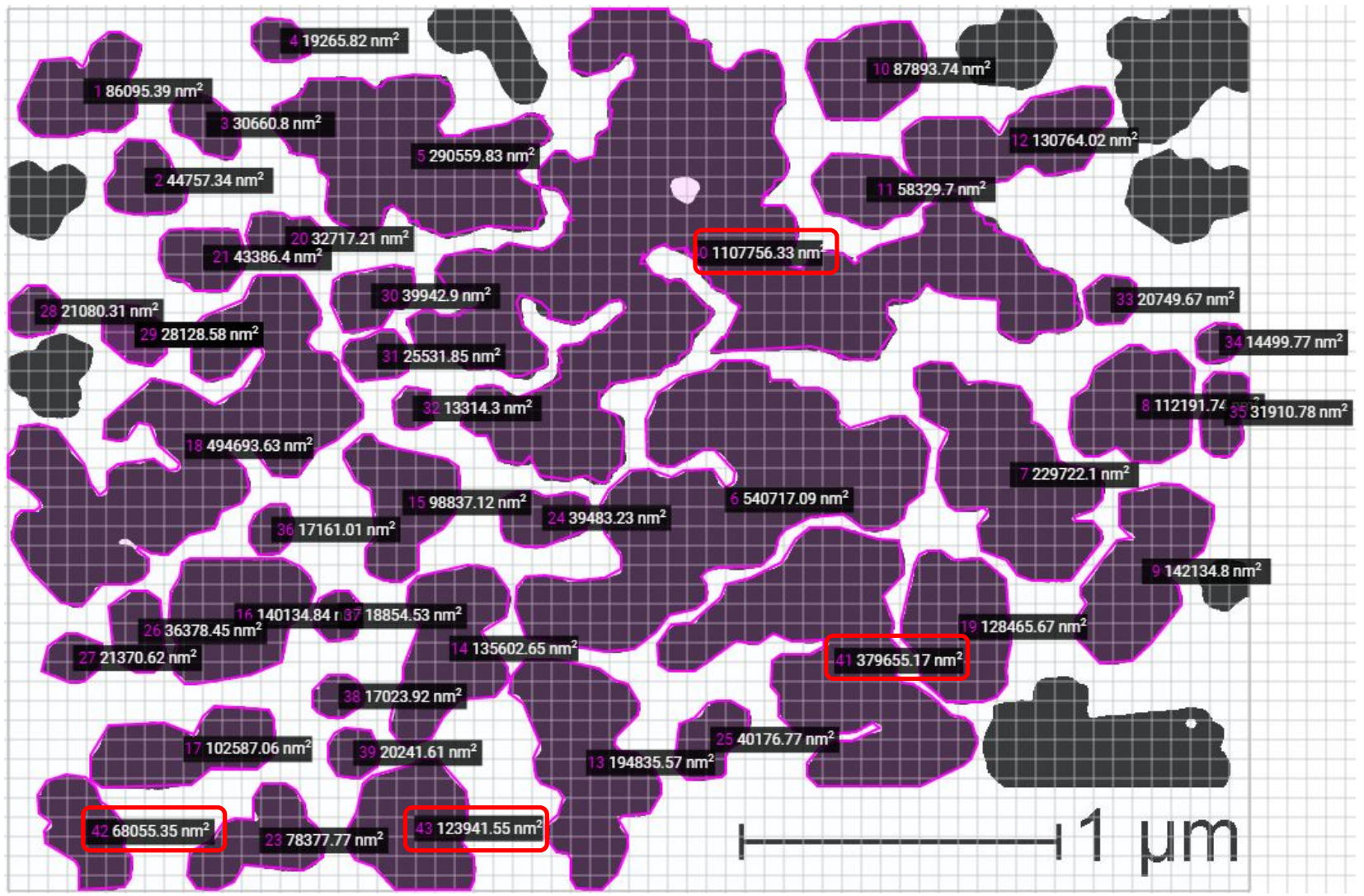


Sample $50.3 \mathrm{M}$ sulfuric acid, $27 \mathrm{~V}, 0-0.5^{\circ} \mathrm{C}$, pre-anodizing duration: 15 hours

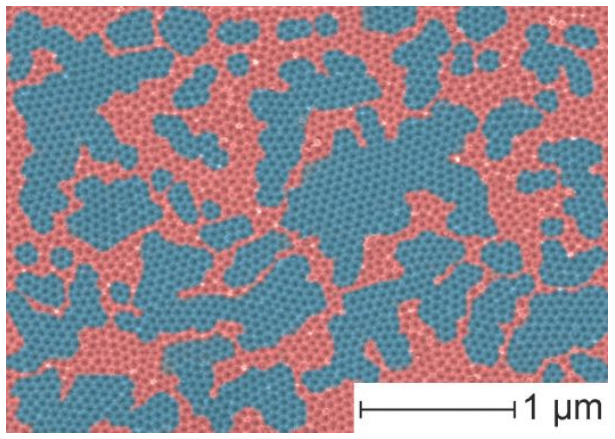

Mean long-range ordered domain size value: $261234 \mathrm{~nm}^{2}$

Standard deviation:

$225981 \mathrm{~nm}^{2}$

Half-width of the confidence interval:

$101611 \mathrm{~nm}^{2}$

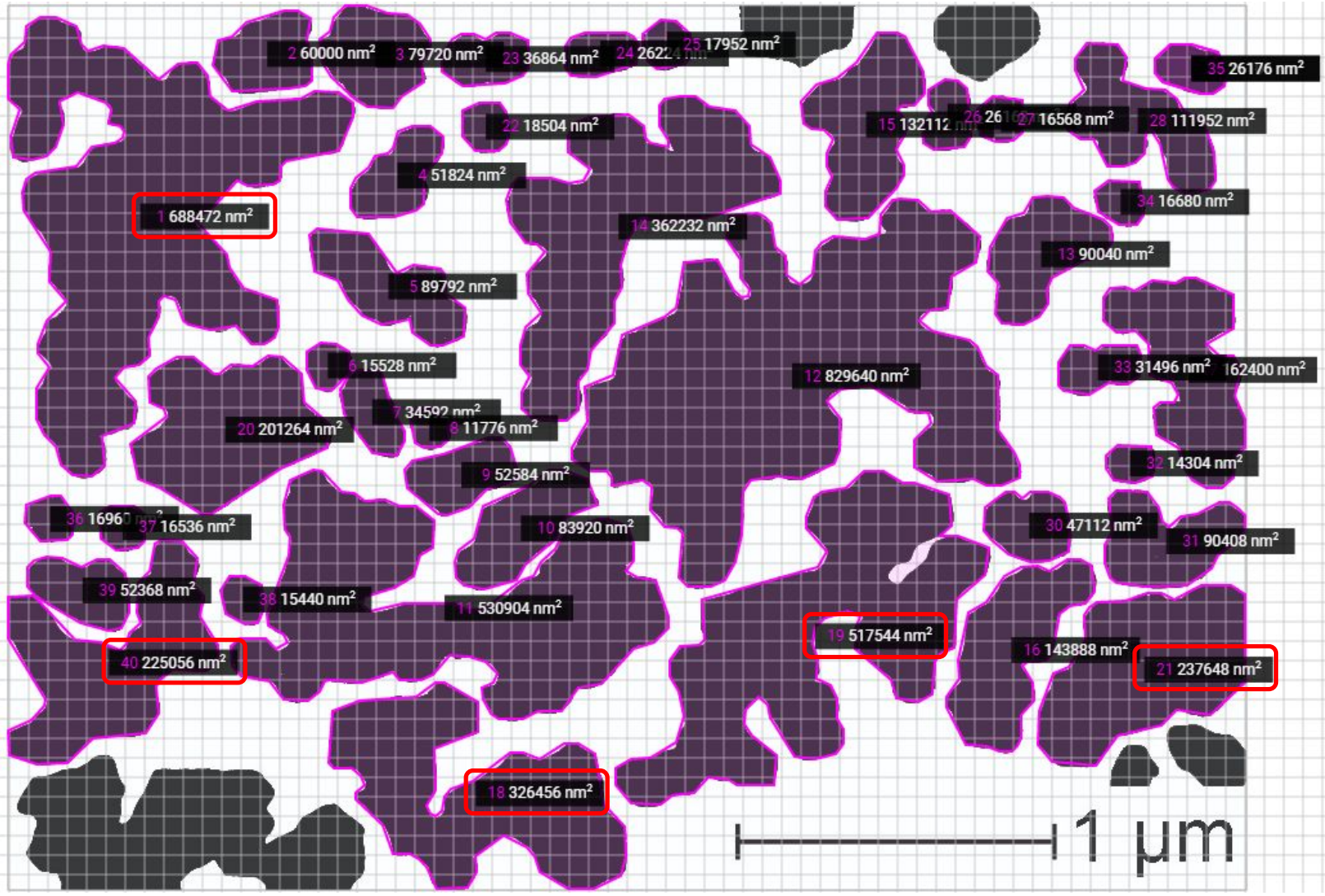


Sample $60.3 \mathrm{M}$ sulfuric acid, $27 \mathrm{~V}, 0-0.5^{\circ} \mathrm{C}$, pre-anodizing duration: 20 hours

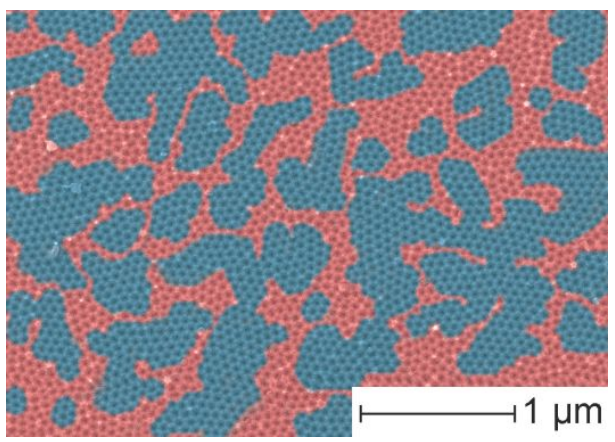

Mean long-range ordered domain size value: $\quad 309827 \mathrm{~nm}^{2}$

Standard deviation:

$369751 \mathrm{~nm}^{2}$

Half-width of the confidence interval:

$175765 \mathrm{~nm}^{2}$

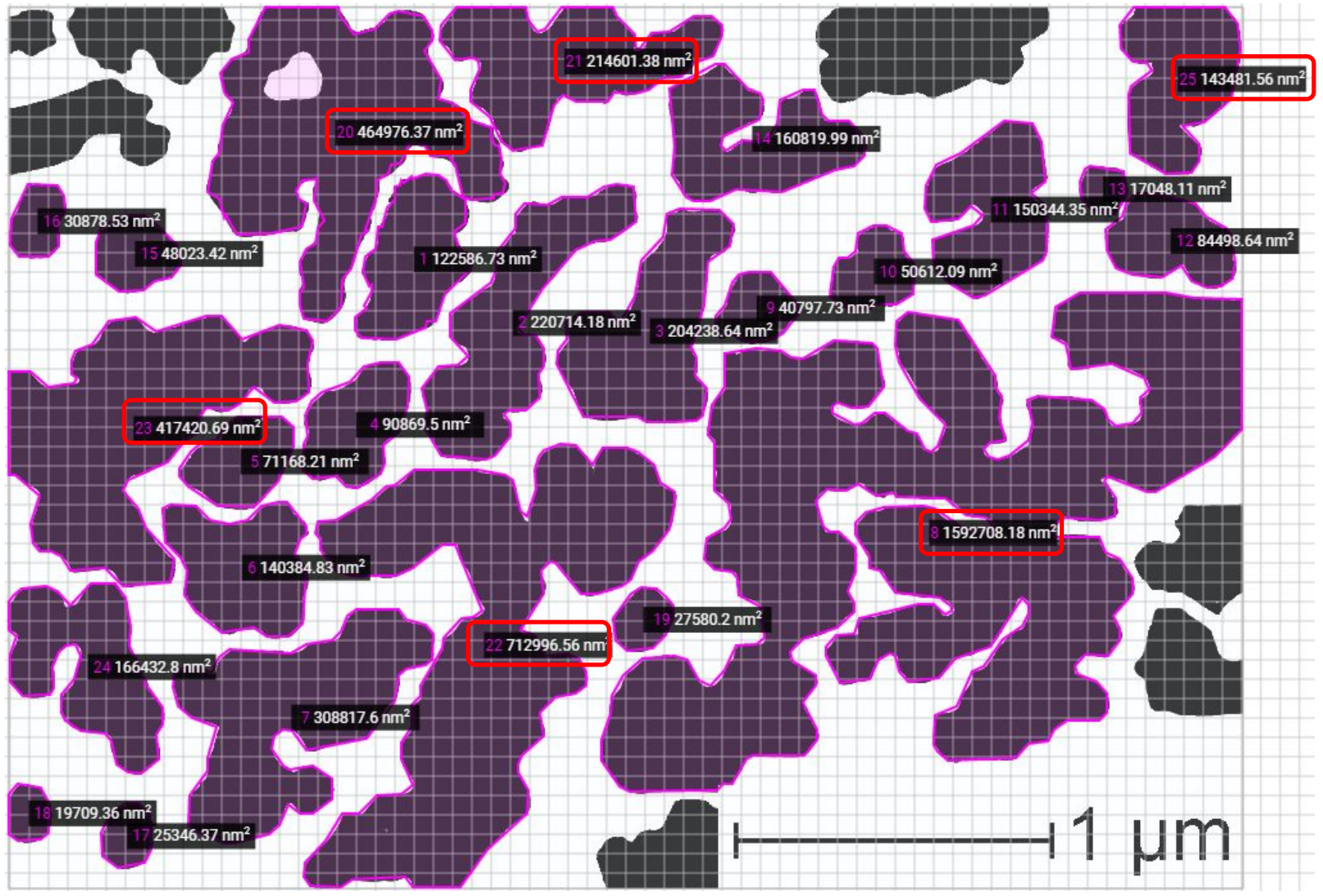


Sample $70.3 \mathrm{M}$ sulfuric acid, $27 \mathrm{~V}, 0-0.5^{\circ} \mathrm{C}$, pre-anodizing duration: 25 hours

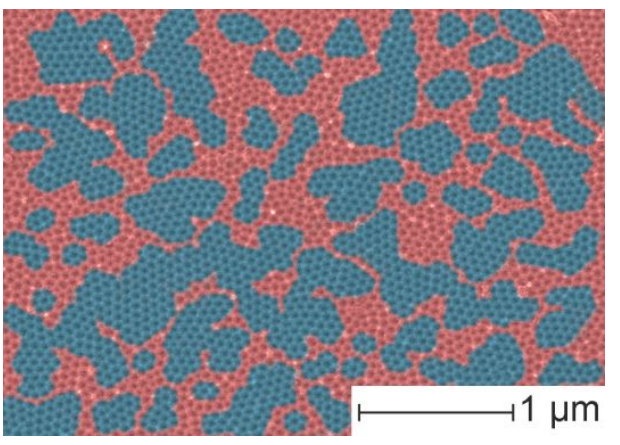

$\begin{array}{ll}\text { Mean long-range ordered domain size value: } & 195558 \mathrm{~nm}^{2} \\ \text { Standard deviation: } & 203734 \mathrm{~nm}^{2} \\ \text { Half-width of the confidence interval: } & 79862 \mathrm{~nm}^{2}\end{array}$

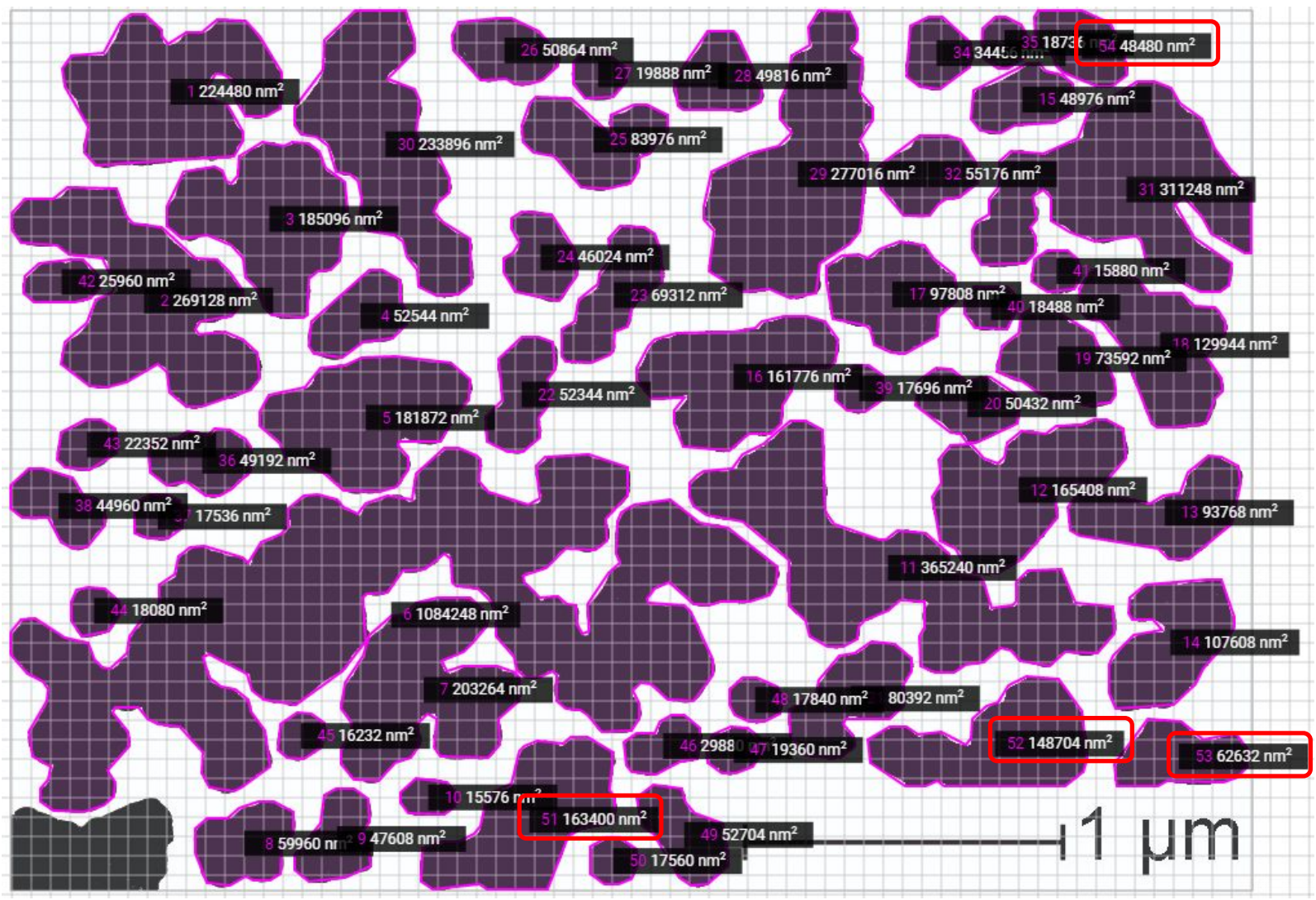


Sample $80.3 \mathrm{M}$ sulfuric acid, $27 \mathrm{~V}, 0-0.5^{\circ} \mathrm{C}$, pre-anodizing duration: 30 hours

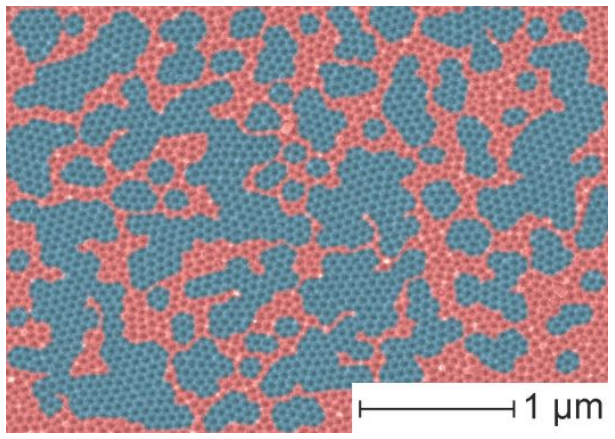

Mean long-range ordered domain size value: $\quad 206274 \mathrm{~nm}^{2}$

Standard deviation:

$222670 \mathrm{~nm}^{2}$

Half-width of the confidence interval:

$87285 \mathrm{~nm}^{2}$

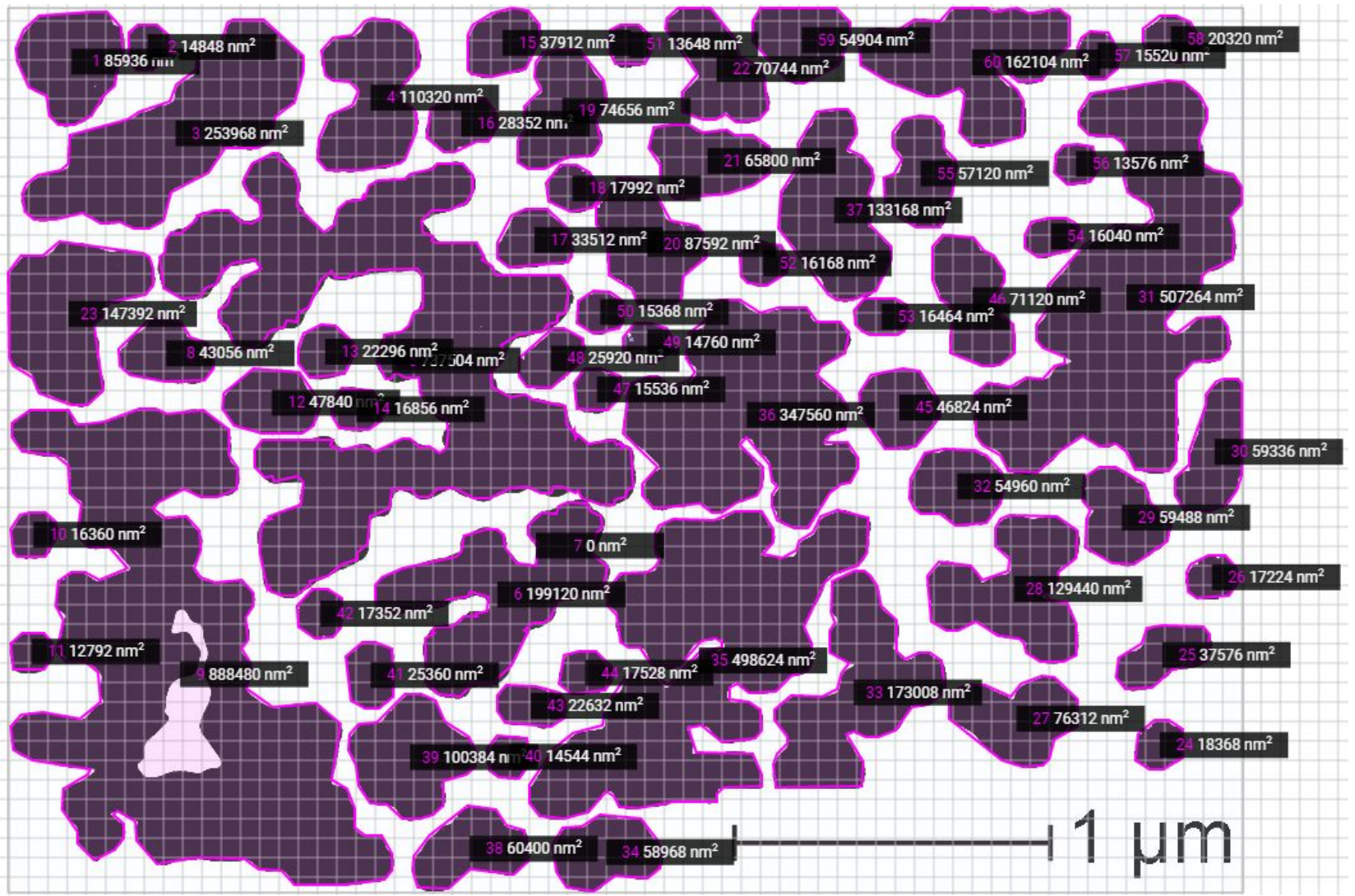


Series of experiments with $0.3 \mathrm{M}$ oxalic acid electrolyte, $40 \mathrm{~V}, 0-0.5^{\circ} \mathrm{C}$ (8 different samples) Threshold used in all cases for dataset refinement and small domains elimination from statistics was $120000 \mathrm{~nm}^{2}$ (approximately corresponds to a symmetrical domain which contains 16 hexagonal cells).

Sample $10.3 \mathrm{M}$ oxalic acid, $40 \mathrm{~V}, 0-0.5^{\circ} \mathrm{C}$, pre-anodizing duration: 0.5 hours

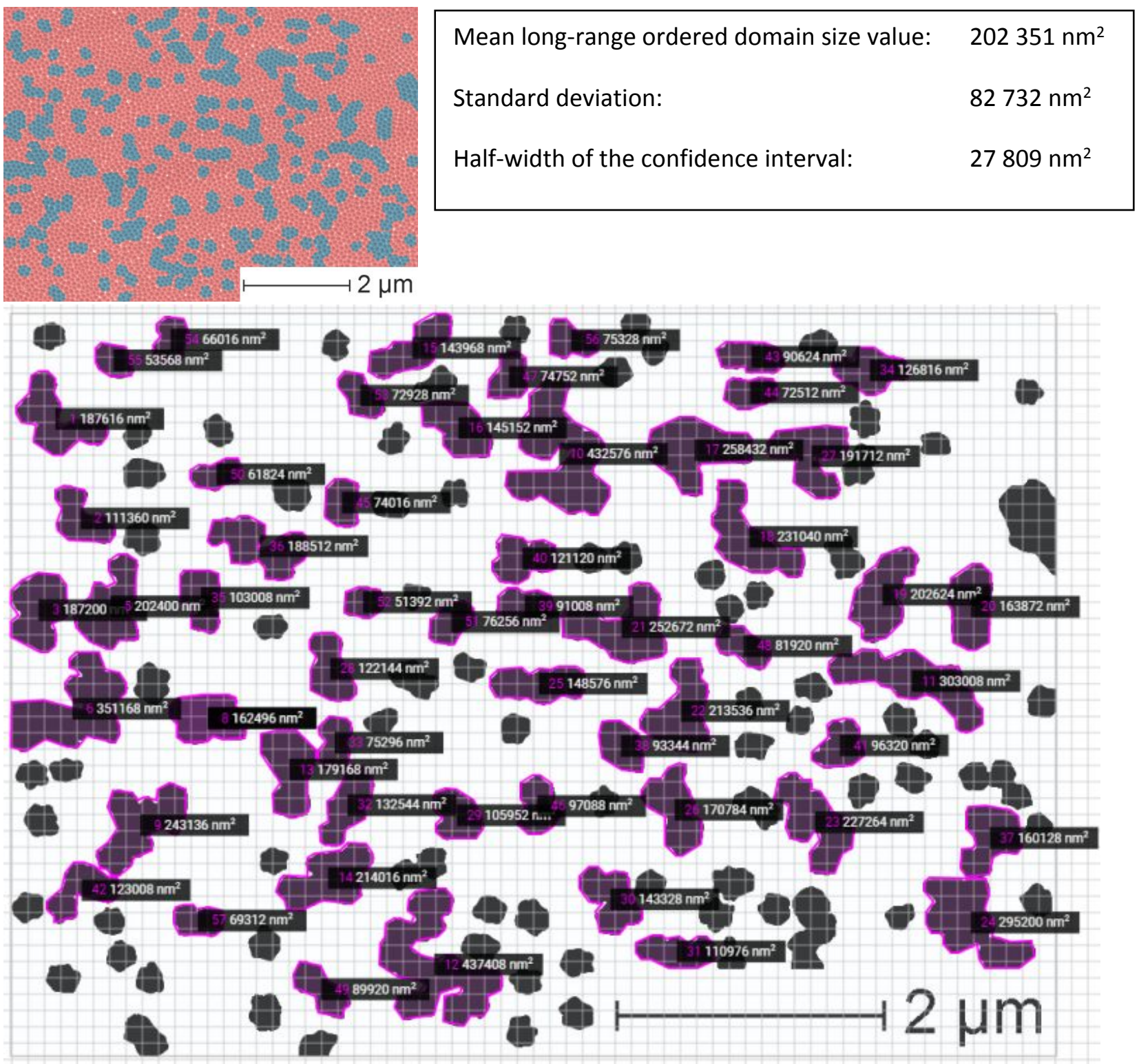


Sample $20.3 \mathrm{M}$ oxalic acid, $40 \mathrm{~V}, 0-0.5^{\circ} \mathrm{C}$, pre-anodizing duration: 1 hour

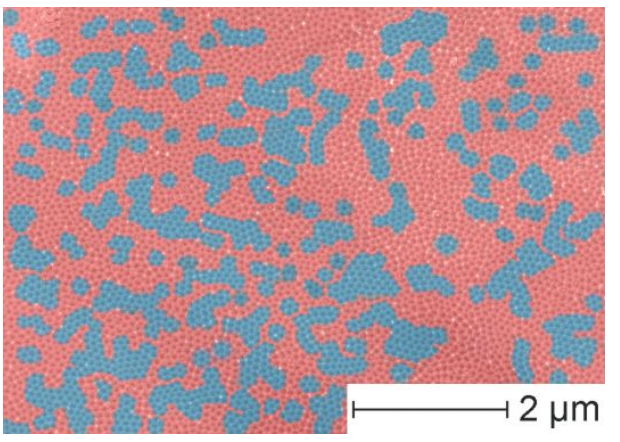

$\begin{array}{lr}\text { Mean long-range ordered domain size value: } & 256624 \mathrm{~nm}^{2} \\ \text { Standard deviation: } & 147619 \mathrm{~nm}^{2} \\ \text { Half-width of the confidence interval: } & 46935 \mathrm{~nm}^{2}\end{array}$

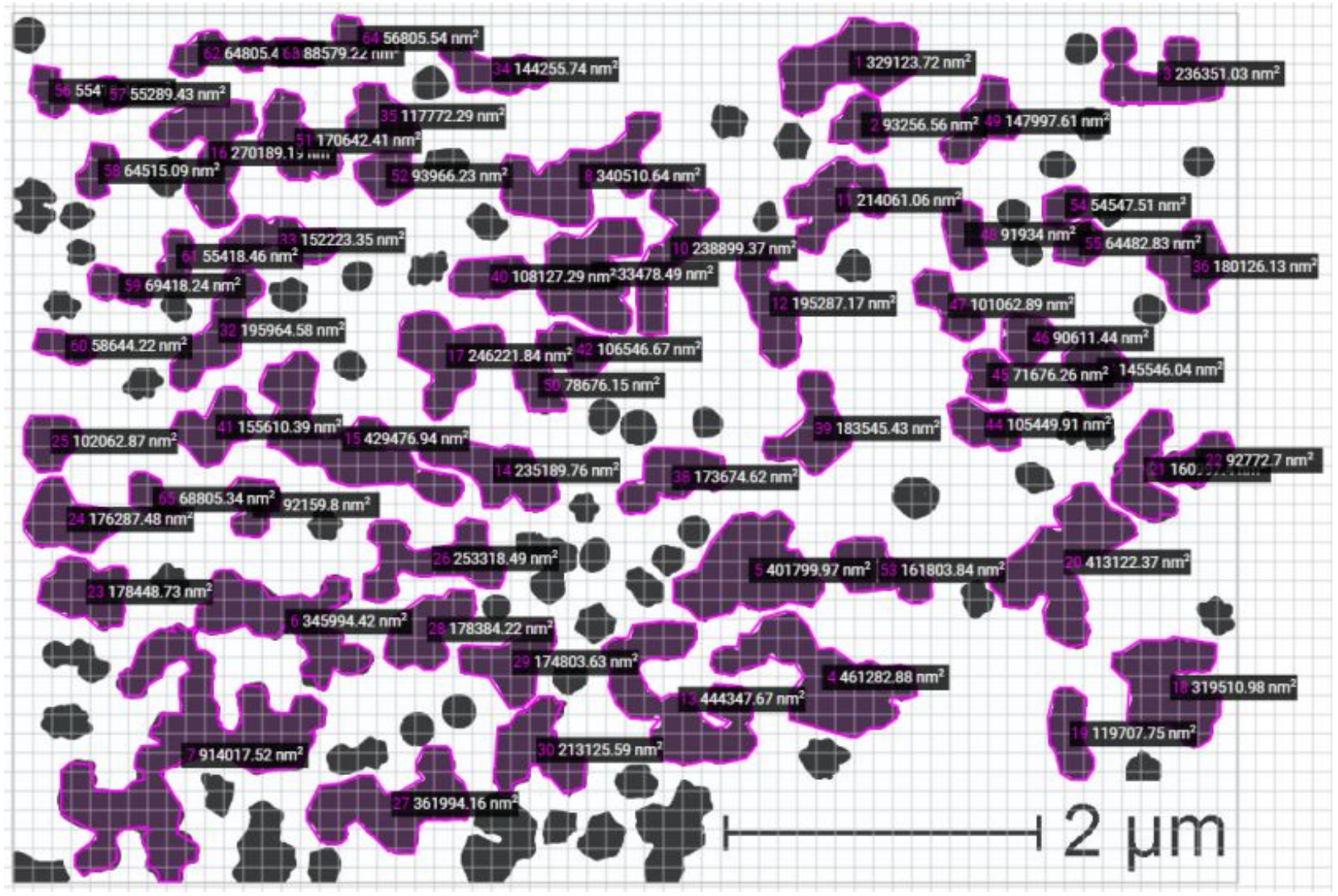


Sample $30.3 \mathrm{M}$ oxalic acid, $40 \mathrm{~V}, 0-0.5^{\circ} \mathrm{C}$, pre-anodizing duration: 5 hours

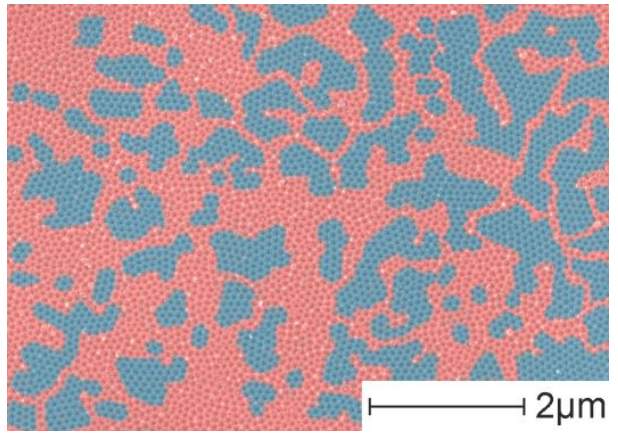

Mean long-range ordered domain size value: $\quad 476178 \mathrm{~nm}^{2}$

Standard deviation:

$364256 \mathrm{~nm}^{2}$

Half-width of the confidence interval:

$134920 \mathrm{~nm}^{2}$

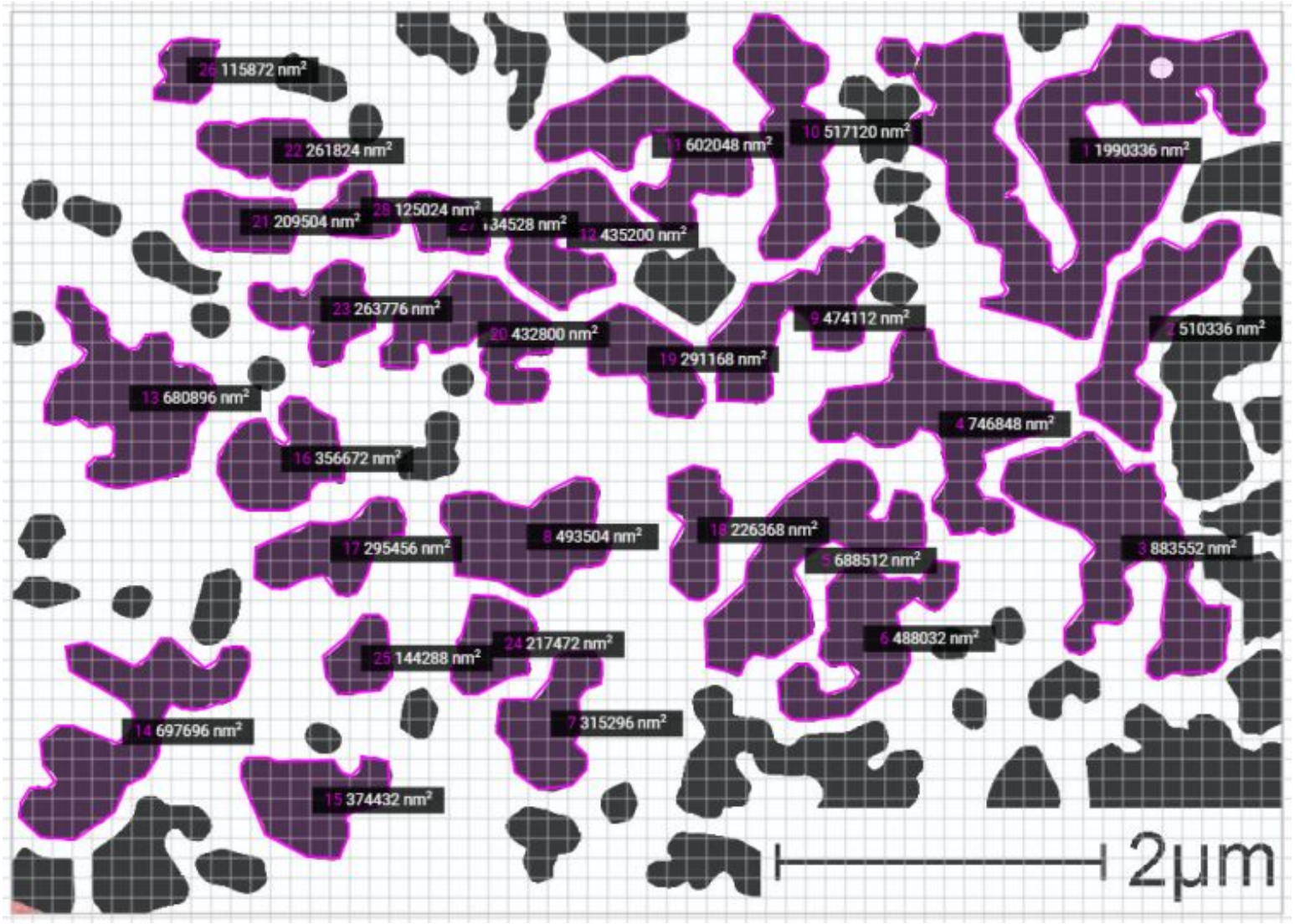


Sample $40.3 \mathrm{M}$ oxalic acid, $40 \mathrm{~V}, 0-0.5^{\circ} \mathrm{C}$, pre-anodizing duration: 10 hours

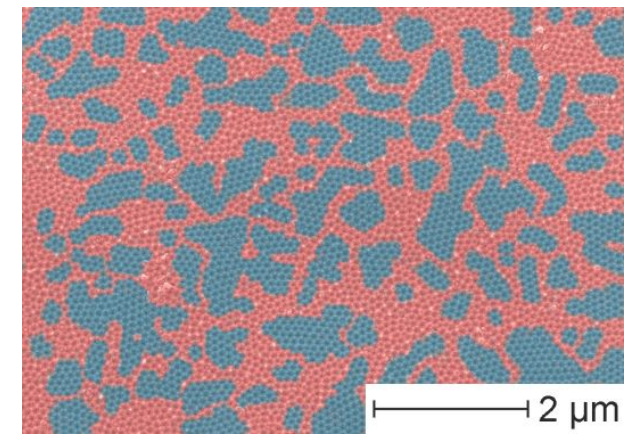

Mean long-range ordered domain size value: $\quad 348103 \mathrm{~nm}^{2}$

Standard deviation:

$359021 \mathrm{~nm}^{2}$

Half-width of the confidence interval:

$114150 \mathrm{~nm}^{2}$

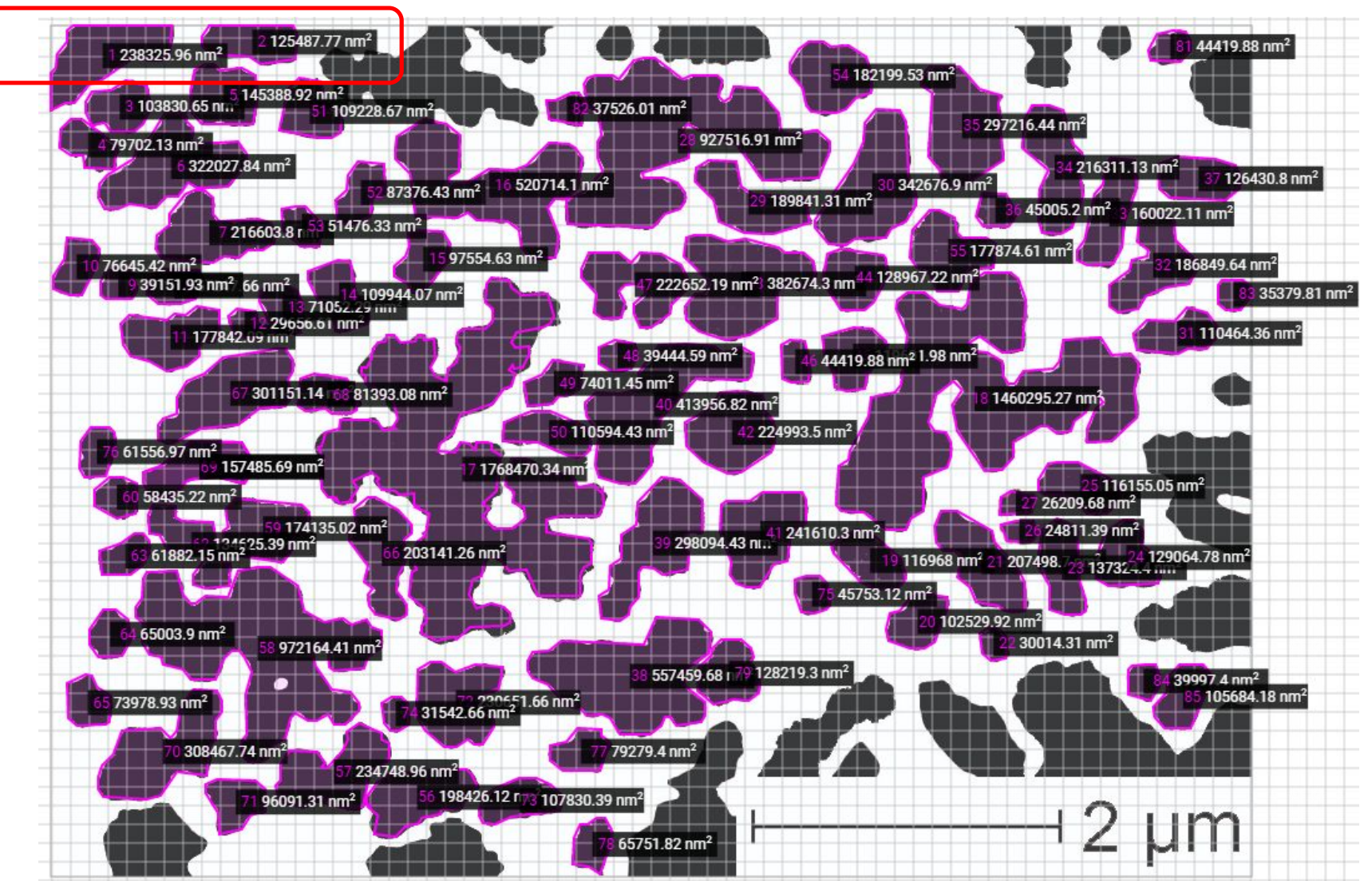


Sample $50.3 \mathrm{M}$ oxalic acid, $40 \mathrm{~V}, 0-0.5^{\circ} \mathrm{C}$, pre-anodizing duration: 15 hours

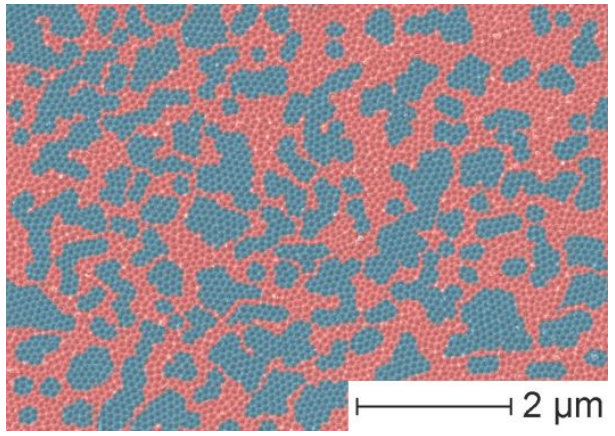

Mean long-range ordered domain size value: $\quad 396018 \mathrm{~nm}^{2}$

Standard deviation:

$418207 \mathrm{~nm}^{2}$

Half-width of the confidence interval:

$128011 \mathrm{~nm}^{2}$

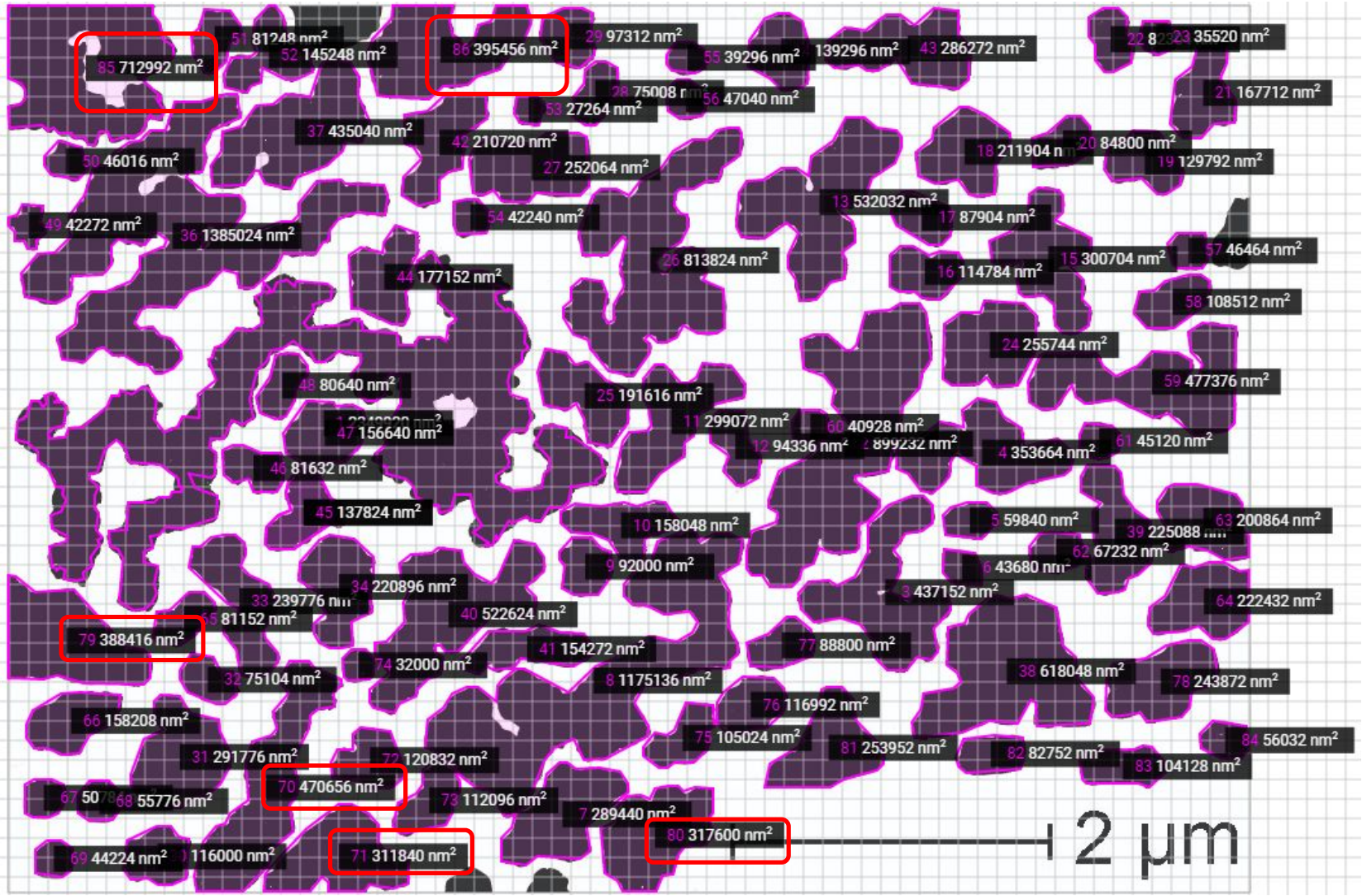


Sample $60.3 \mathrm{M}$ oxalic acid, $40 \mathrm{~V}, 0-0.5^{\circ} \mathrm{C}$, pre-anodizing duration: 20 hours

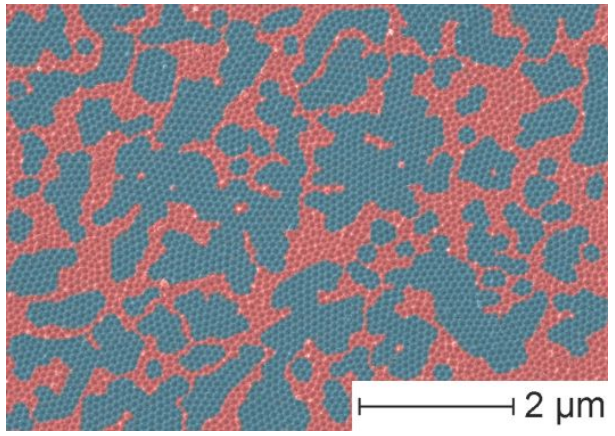

Mean long-range ordered domain size value: $\quad 577182 \mathrm{~nm}^{2}$

Standard deviation:

$871426 \mathrm{~nm}^{2}$

Half-width of the confidence interval:

$311830 \mathrm{~nm}^{2}$

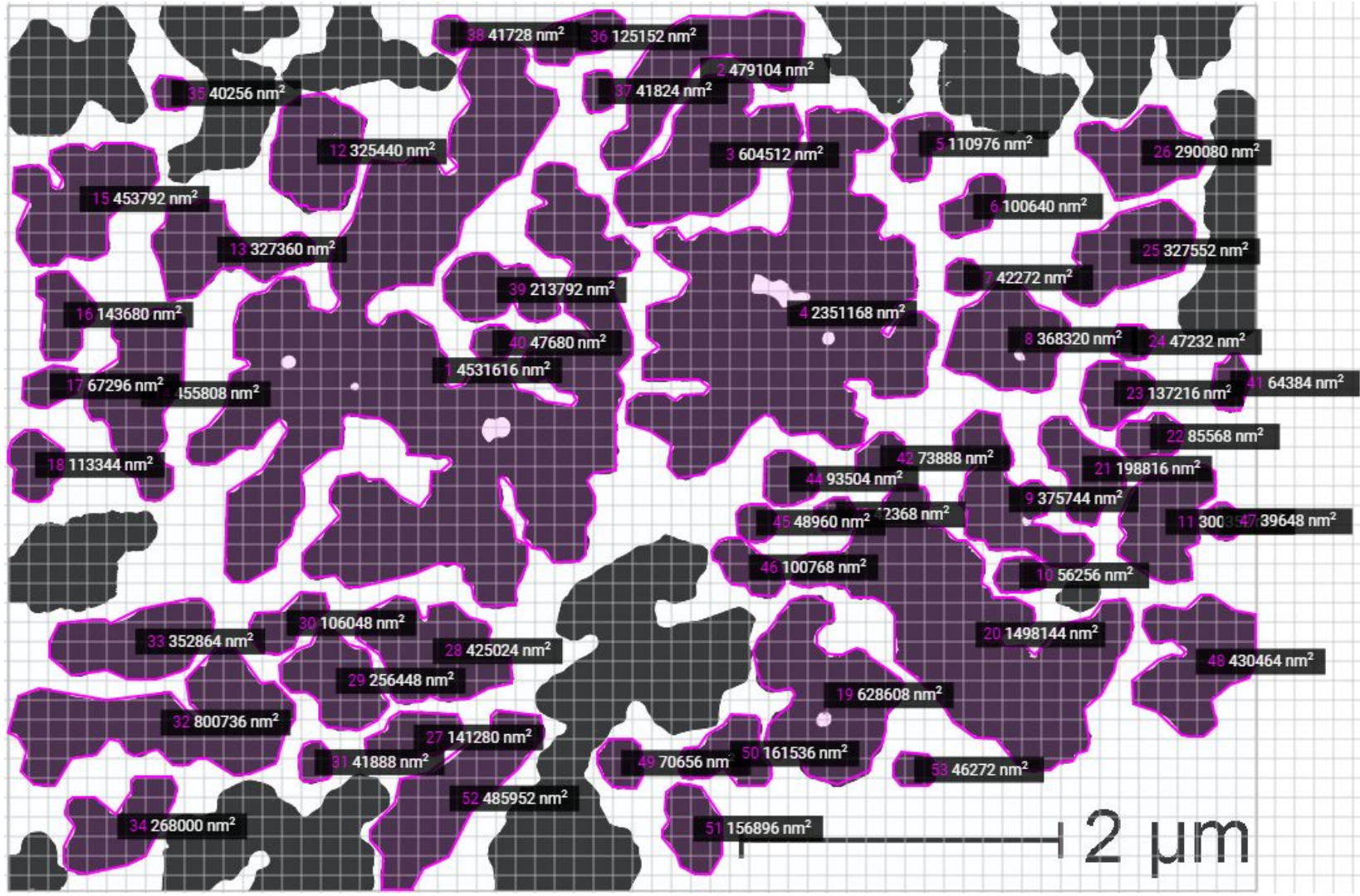


Sample $70.3 \mathrm{M}$ oxalic acid, $40 \mathrm{~V}, 0-0.5^{\circ} \mathrm{C}$, pre-anodizing duration: 25 hours

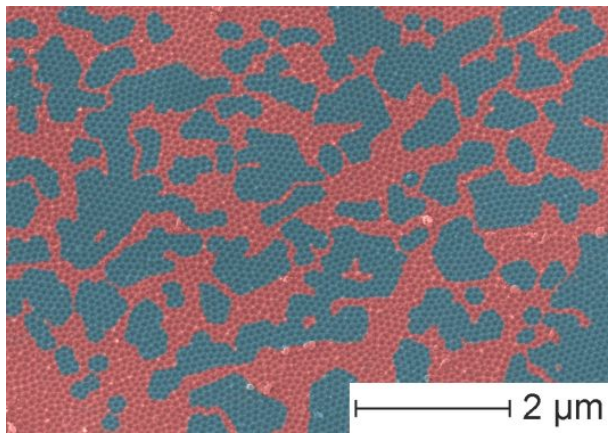

Mean long-range ordered domain size value: $\quad 487564 \mathrm{~nm}^{2}$

Standard deviation:

$549098 \mathrm{~nm}^{2}$

Half-width of the confidence interval:

$190249 \mathrm{~nm}^{2}$

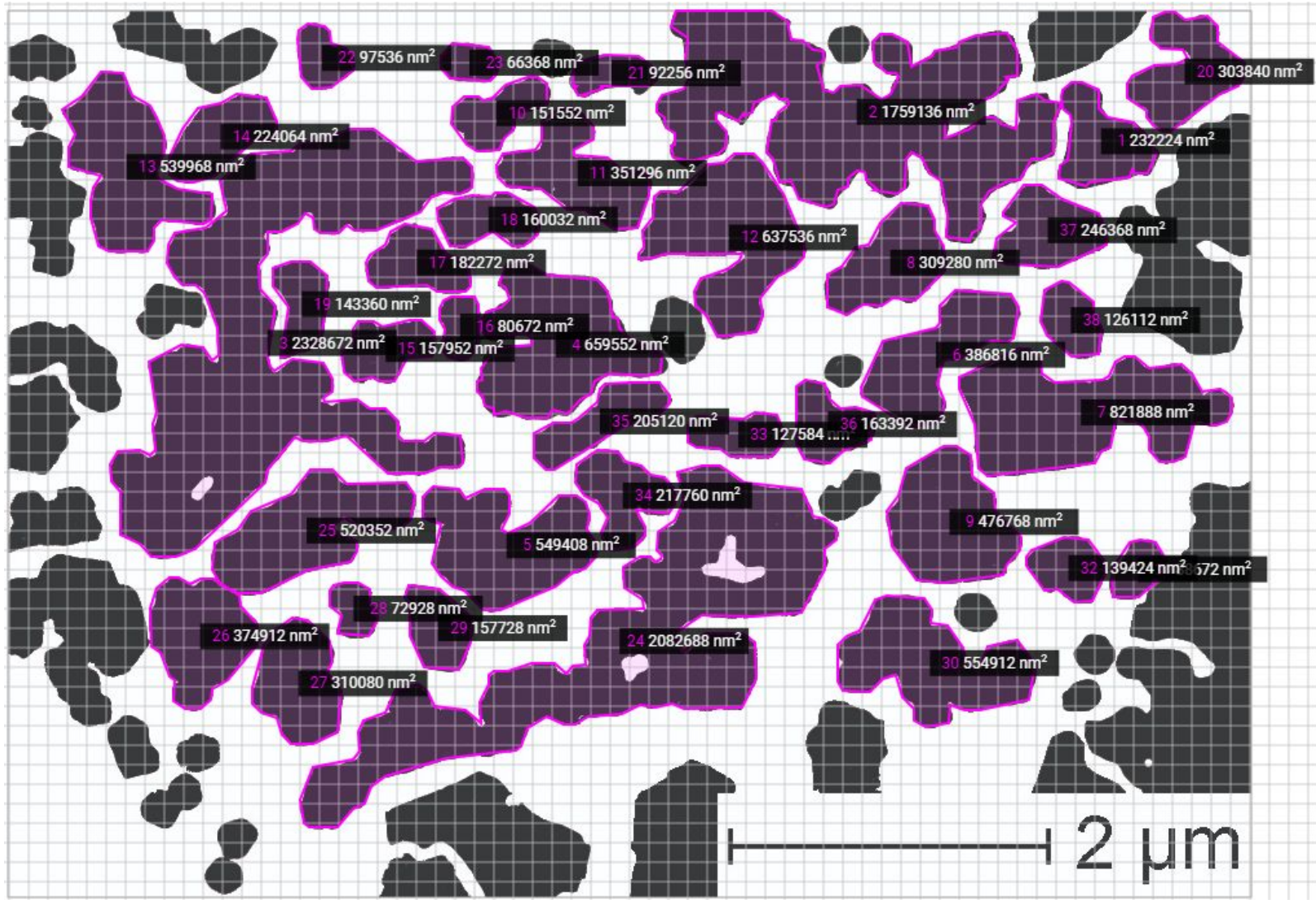


Sample $80.3 \mathrm{M}$ oxalic acid, $40 \mathrm{~V}, 0-0.5^{\circ} \mathrm{C}$, pre-anodizing duration: 30 hours

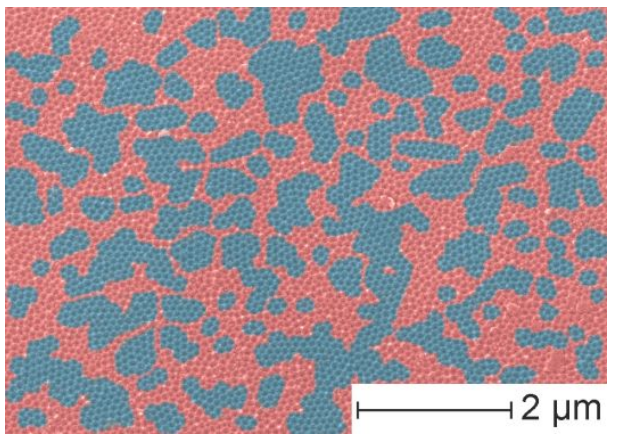
Mean long-range ordered domain size value:
$289277 \mathrm{~nm}^{2}$
Standard deviation:
$178349 \mathrm{~nm}^{2}$
Half-width of the confidence interval:
$53307 \mathrm{~nm}^{2}$

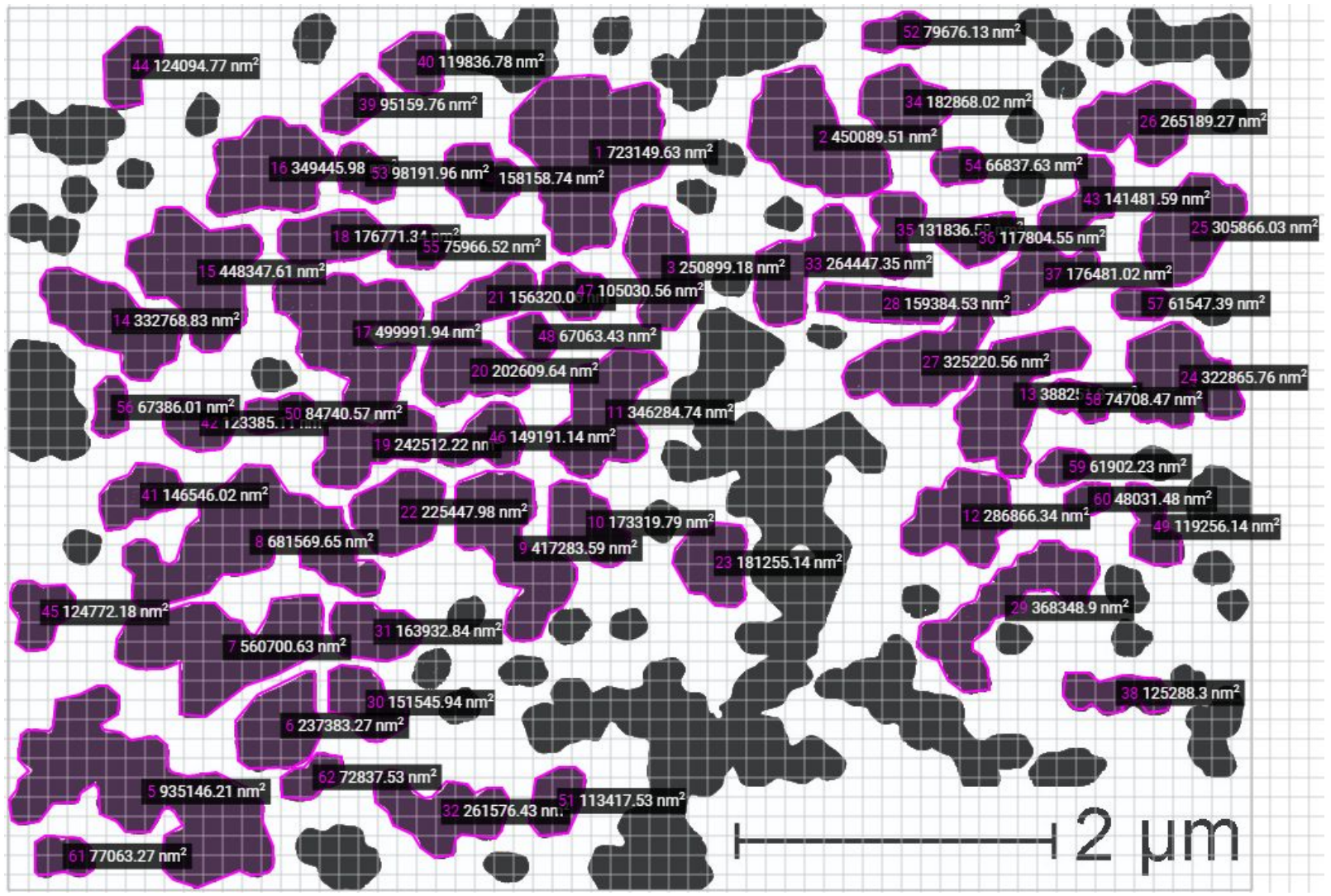




\section{Calculation of the analytical enhancement factors of different SERS substrates}

Analytical enhancement factors were calculated according to the equation

$$
A E F=\frac{I_{S E R S} / c_{S E R S}}{I_{R S} / c_{R S}}
$$

where $\mathrm{C}_{\text {SERS }}$ and $\mathrm{I}_{\text {SERS }}$ are the concentration $\left(1 \times 10^{-6} \mathrm{M}\right)$ and SERS intensity for the SERS measurement on the SERS substrate under investigation, respectively, and $C_{R S}$ and $I_{R S}$ are the concentration $\left(1 \times 10^{-3} \mathrm{M}\right)$ and Raman intensity for the regular Raman measurement of RhB on a glass substrate, respectively. For the Raman and SERS signal the most intense peak at $1645 \mathrm{~cm}^{-1}$ at an excitation wavelength of $532 \mathrm{~nm}$ was used where the peak heights were used for the intensity.

Table S1. Calculated analytical enhancement factors for different sulfuric and oxalic PAA-based SERS substrates presented in this work.

\begin{tabular}{|c|c|}
\hline Anodization time & Enhancement factor \\
\hline \multicolumn{2}{|c|}{ Sulfuric acid PAA + $5 \mathrm{~nm} \mathrm{Ag}$} \\
\hline $0.5 \mathrm{~h}$ & $6.54 \times 10^{4}$ \\
\hline $10 \mathrm{~h}$ & $9.43 \times 10^{4}$ \\
\hline $20 \mathrm{~h}$ & $1.54 \times 10^{5}$ \\
\hline $30 \mathrm{~h}$ & $9.08 \times 10^{4}$ \\
\hline \multicolumn{2}{|c|}{ Oxalic acid PAA + $5 \mathrm{~nm} \mathrm{Ag}$} \\
\hline $0.5 \mathrm{~h}$ & $2.12 \times 10^{3}$ \\
\hline $10 \mathrm{~h}$ & $3.71 \times 10^{3}$ \\
\hline $20 \mathrm{~h}$ & $8.13 \times 10^{3}$ \\
\hline $30 \mathrm{~h}$ & $5.16 \times 10^{3}$ \\
\hline
\end{tabular}


